\title{
Computer optimization of a minimal biped model discovers walking and running
}

\author{
Manoj Srinivasan and Andy Ruina \\ Biorobotics and Locomotion Laboratory \\ Cornell University, Ithaca, NY 14853 USA \\ Available Online at Nature.com: Sept 11, 2005. \\ In Print. Nature. 439, 72-75, Letters: Jan 05, 2006.
}

Note: This is a "pre-print" version of the paper published in Nature. It is word-for-word the same as the published version, except where noted explicitly as footnotes. Five minor typographical errors in the published version have been corrected in this version, as also a small error in Figure 3. None of the conclusions of the paper are affected by these changes.

Although people's legs are capable of a broad range of muscle-use and gait patterns, they generally prefer just two. They walk, swinging their body over a relatively straight leg with each step, or run, bouncing up off a bent leg between aerial phases. Walking feels easiest when going slowly, and running feels easiest when going faster. More unusual gaits seem more tiring. Perhaps this is because walking and running use the least energy $[1 ; 2 ; 3 ; 4 ; 5$; 6; 7]. Addressing this classic [1] conjecture with experiments $[2 ; 3]$ requires comparing walking and running with many other strange and unpracticed gaits. As an alternative, a basic understanding of gait choice might be obtained by calculating energy cost by using mechanics-based models. Here we use a minimal model that can describe walking and running as well as an infinite variety of other gaits. We use computer optimization to find which gaits are indeed energetically optimal for this model. At low speeds the optimization discovers the classic inverted-pendulum walk $[8 ; 9 ; 10 ; 11 ; 12 ; 13]$, at high speeds it discovers a bouncing run $[12 ; 14]^{1}$, even without springs, and at intermediate speeds it finds a new pendular-running gait that includes walking and running as extreme cases.

One way of characterizing gaits is by the motions of the body (Fig. 1a). In these terms, walking seems well caricatured [13] (Fig. 1b) by the hip joint going from one circular arc to the next with push-off and heelstrike impulses in between. Similarly, running could be caricatured by a sequence of parabolic free-flight arcs (Fig. 1c), with impulses from the ground at each bounce $[14 ; 15 ; 16]$.

Why do people not walk or even run with a smooth level gait [8], like a waiter holding two cups brim-full of boiling coffee? Why do people select walking and running from the other possibilities? We address such questions by modeling a person as a machine describable with the equations of newtonian mechanics. The basic approximations are: first, that humans have compact bodies and light legs; second, that gait choice is based on energy optimization $[1 ; 4]$; and third, that energy cost is proportional to muscle work $[2 ; 4 ; 8]$. We use a simplification of previous models $[4 ; 6 ; 7]$, perhaps the simplest mechanical model that is capable of exhibiting a broad range of gaits that includes walking and running. Although the model is a mechanical abstraction that is not physically realizable, it is subject to the laws of physics. Because of its simplicity, the model is amenable to interpretation. It can also be studied with exhaustive and accurate simulation experiments, far beyond what is possible with human subjects.

We wish to find how a person can get from one place to another with the least muscle work $W$ (Methods). We treat the body as a point mass $m$ at position $(x, y)$ at time $t$ (Fig. 2a). The legs are massless and therefore, when not in ground contact, they can be oriented, lengthened and shortened with no energy cost. The fluctuations of the leg length $l(t)$ due to flexion of the hip, knee and ankle are incorporated in a single telescoping axial actuator [4] that carries a compressive time-varying force $F=F(t)$. For simplicity, we seek

\footnotetext{
${ }^{1}$ The published version incorrectly referred to $[12 ; 13]$
} 
a) Some possible gaits

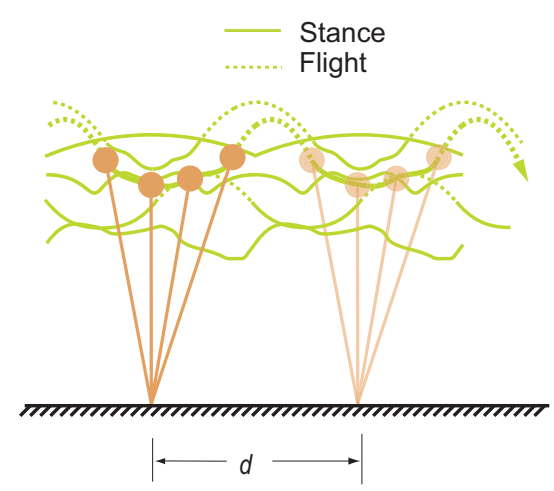

c) Impulsive run

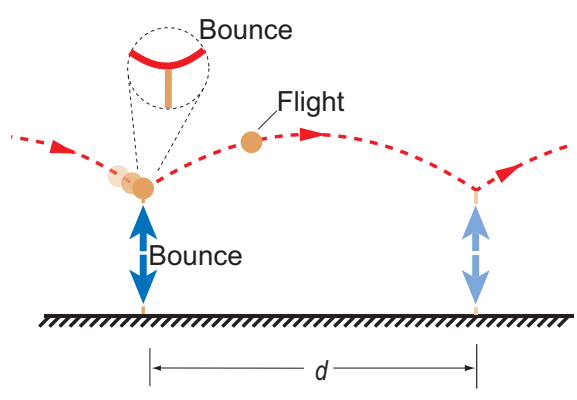

b) Inverted pendulum walk

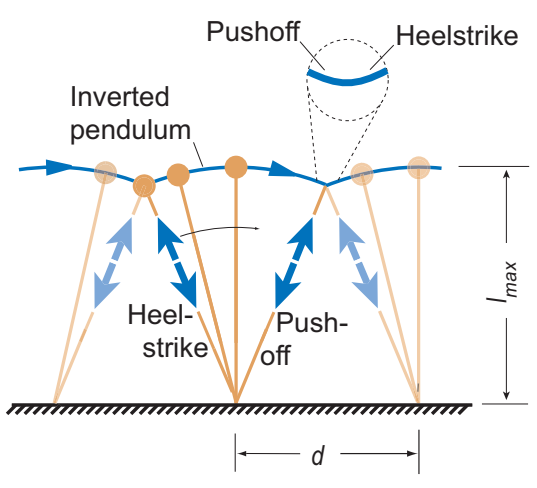

d) Hybrid intermediate gait: pendular run

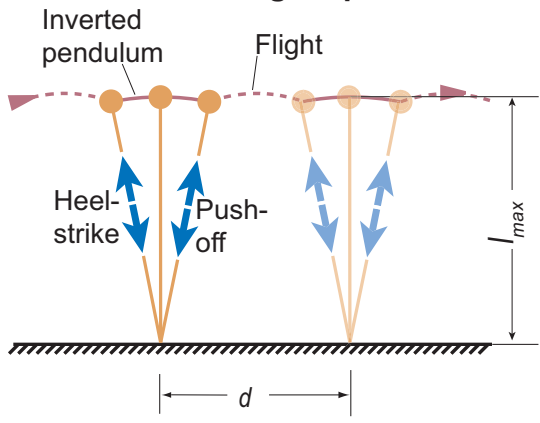

Figure 1: Body motion in human gaits. a, Trajectories of the center of mass for a few possible gaits. Solid lines, stance; dotted lines, flight. b, Trajectory for inverted-pendulum walking. c, Trajectory for impulsive running. d, Trajectory for a new gait: pendular running. At least one of the gaits $\mathbf{b}, \mathbf{c}$ and $\mathbf{d}$ turns out to use less work than any other candidates (for example, from a, according to the calculations here.

an explanation of gait choice with no essential dependence on elastic energy storage; we assume no springs (tendons) in series or parallel with the actuators.

We assume that during the stance phase, when a foot is in contact with the rigid level ground, that it does not slip. At most one foot can be in contact with the ground at a time. During stance, both gravity $m g$ and $F$ act on the body (Fig. 2a). During the flight phase, when neither leg touches the ground, gravity is the only force. We seek periodic motions, in which each step is like the previous step. The left and right legs have identical force and length profiles. A single step consists of one stance phase (possibly short, as in high-speed running) and one flight phase (possibly of zero duration, as in walking).

A gait is characterized by the position and velocity of the body at the start of a stance phase relative to the stance foot, by the step period, and by $F(t)$. Given these, we can integrate the newtonian equations of motion forwards in time to find the body trajectory and leg length as functions of time (including the maximum leg length $\left.l_{\max }\right)$. At the end of the step, we assume that the next foot is placed on the ground at the same position relative to the body as at the start. We can thus calculate the step length $d$, the average forward speed $v$, and the work done by the leg per unit weight and distance $C=W /(m g d)$. For random $F(t)$, the final body height and velocity generally do not match the starting conditions and therefore do not generate a periodic gait. Nonetheless, by appropriately varying $F(t)$ we can find infinitely many periodic gaits (Fig. 1a) with all manner of complicated trajectories (Methods). Of those periodic gaits, we wish to find those that minimize the cost $C$.

The optimal solutions have cost arbitrarily close to zero unless the optimization is further constrained. The cost can be made arbitrarily small by growing the leg length (and the locomotion becomes akin to the rolling of a giant multi-spoked wheel), so we set the maximum length to be $l_{\max }$, representing the leg length. Because we have no leg-swing cost, $C$ can be reduced to zero by taking very small steps $[6 ; 12 ; 17]$ so we optimize for various fixed values of step length $d$. Finally, $C$ has a non-anthropomorphic lower bound (corresponding to standing on one leg for an infinite time mid-step), approached as the average speed $v$ goes to zero, so we constrain $v$.

After non-dimensionalizing using $m, g$ and $l_{\max }$, 
no free parameters remain. We seek solutions as two conditions are varied: the dimensionless average speed $^{2} V=v / \sqrt{g l_{\max }}\left(V^{2}\right.$ is the so-called Froude number) and the dimensionless step length $D=$ $d / l_{\max }$. For given values of $V$ and $D$, the optimal periodic gait is determined with numerical optimal control methods that are more or less standard (Methods).

All optimizations converged toward one of three stereotypical collisional gaits, depending on $V$ and $D$, but never to a smooth collisionless gait. First, at low $V$, the classic inverted-pendulum walking gait (Figs. 1b and $2 \mathrm{~b}$ ) is optimal. Second, at high $V$, an impulsive running gait is optimal (Figs 1c and 2c). Third, at intermediate $V$, a new gait, pendular running (Figs. 1d and $2 \mathrm{~d}$ ), is optimal. Pendular running has a flight phase between extended inverted-pendulum stance phases. Pendular running is a generalization of, and a connection between, walking and running: with no flight phase it is inverted-pendulum walking; with an infinitesimal pendular phase it is impulsive running.

The numerical optimization, unbiased by an expectation of what the optimal gaits might be, has thus discovered the classic gaits that caricature walking and running. The new third gait might be the model's way of running with a non-zero stance phase, given the model's lack of tendons. A tentative prediction would be the existence of a ground force versus time curve with two humps during the stance phase for, perhaps, weak or obese people running slowly. The respective regions of optimality of the three gaits are shown in Fig. 3.

Alexander $[8 ; 10]$ argued that inverted-pendulum walking is limited to those speeds at which the centripetal acceleration of a body pivoting over a straight leg is less than gravity, ensuring that the body does not vault off the ground. However, walking becomes energetically non-optimal at speeds lower than the above limit $[8 ; 10]$ (Fig. $3^{3}$ ). Indeed, people switch from a walk to run $[18 ; 19]$ at about $\mathrm{V}=0.65$ and $\mathrm{D}=0.95$, close to the boundary at which walking ceases to be optimal (Fig. 3) in this model.

The numerical optimization results are buttressed by heuristic considerations. The cost $C$ is an integral of the leg power (Methods). There are two ways of setting this power to zero: setting $i=0$ (corresponding to inverted-pendulum motion) or setting $F=0$ (corresponding to free flight). Thus, the flight phase $(F=0)$ of running is an energy-saving analogue of the pendu$\operatorname{lar}(i=0)$ motion of walking; both phases involve no work. All the work is crowded into brief impulses at appropriate times.

Inverted-pendulum walking, pendular running and impulsive running all have work-free motions, punctuated by impulses (collisions). The costs of these collisional gaits can be calculated directly $[10 ; 11 ; 12]$. For inverted-pendulum walking, positive work performed during push-off is evaluated as the difference in kinetic energy just before and after the push-off $[8 ; 20$; 12]. $C_{\text {walking }}=D V_{I}^{2} /\left(8-2 D^{2}\right)$, where $V_{I}$ is the magnitude of the velocity vector just before pushoff. For impulsive running, cost is equal to the vertical kinetic energy that is lost and regained in every bounce $[12 ; 13]\left(C_{\text {running }}=D / 8 V^{2}\right)$. For a given $V$ and small values of $D$, the cost for the collisional gaits is proportional [12] to the square of the kink-angle in the trajectory (Fig. 4c). The energetic trade-off between inverted-pendulum walking and impulsive running (Fig. 4a, b) can be understood as a minimization of collision angles [12] for a specific step length $D$. At low speeds the circular arc of walking has shallower collisions than the parabolic free-flight of running, and at high speeds the situation is reversed (Fig. 4c).

The optimizations here show that smooth collisionless gaits require more work than the optimal collisional gaits. For example, consider a flat walk $[8 ; 10]$, in which the body moves at constant height. This gait has $[8 ; 10] C_{\text {flat }}=D / 8 \sqrt{1-D^{2}}$. Figure $4 \mathrm{a}$, b shows that the exceptionally smooth, flat walk is never optimal (Methods). Recent human experiments [21; 22] also show that a flat walk uses more energy than normal walking.

As has been found for a gait model that assumes collisions a priori [12], the more general model here shows that it is advantageous to simulate elasticity during running, even with no genuine elasticity (tendons). Indeed, real human legs do approximately simulate an elastic spring during running [16; 23]. More generally, the model here, as well as simpler models $[4 ; 8 ; 12]$, indicates that the energetic utility of running probably does not depend on genuine elasticity in the legs. However, such elasticity, neglected here, would further decrease the cost of running $[4 ; 6 ; 9]$, supporting the idea [24] that human ancestors could have started to run before the modern human long Achilles tendon was fully evolved.

To maximize simplicity of calculation and interpretation, we have neglected various crucial features including a cost for leg-swing $[12 ; 17 ; 26]$, a more realistic model of muscle cost [7;27], allowance of a non-infinitesimal double-stance phase $[4 ; 6 ; 28]$, elas-

\footnotetext{
${ }^{2}$ The published version had $V=v / \sqrt{g l / \max }$, a typo.

${ }^{3}$ In Fig. 3 of the published version, the y-intercept of the dashed line was at about 1.7. The y-intercept should be at about 1.5 as shown in Fig. 3 in this corrected version.
} 


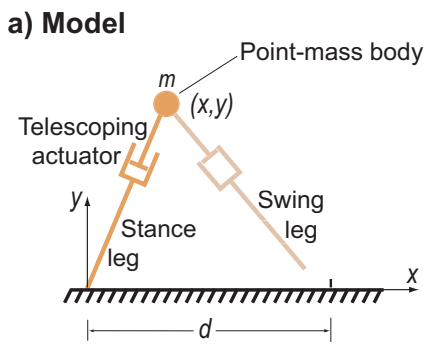

b) Pendular walk

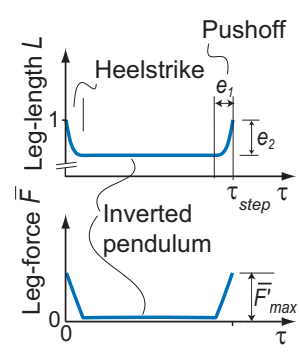

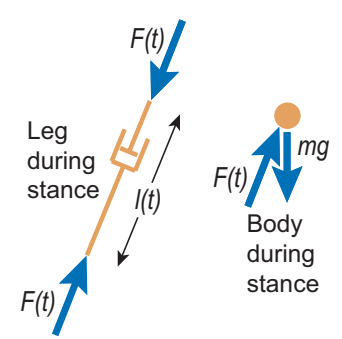

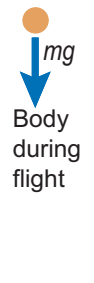

c) Impulsive run

d) Pendular run

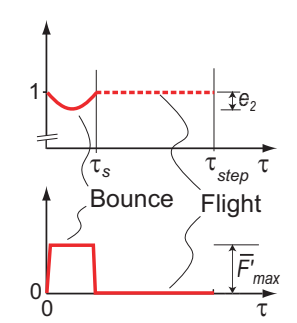

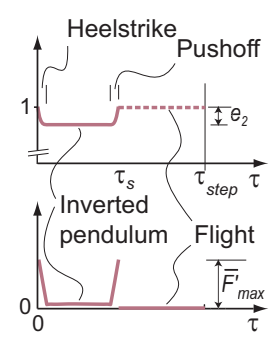

Figure 2: Point-mass biped model and its optimal solutions. a, The configuration shown is part way through the stance phase. The next stance leg is oriented to prepare for a new contact at a distance $d$ from the last. b-d, Dimensionless force and length shown as functions of dimensionless time, for the three optimal gaits (b, pendular walk; $\mathbf{c}$, impulsive run; $\mathbf{d}$, pendular run), before full convergence of the numerical optimization. The finite forces in the figures are approximations to the converged impulsive (collisional) forces. In the extrapolated optimum, as the grid size $h \rightarrow 0$ and the allowed force upper bound $F_{\max }$, the optimizations find that $e_{1}, e_{2} \rightarrow 0$ and that the maximum forces used go to infinity (Methods). In these limits the walking gait (b) is an inverted pendulum with heel-strike and push-off impulses, the running gait (c) is an impulsive bounce between free flights, and the pendular run (d) has constant-length pendulum phases and flight phases separated by impulses.

tic and dissipative elements in series with the actuator $[4 ; 6 ; 7 ; 24]$, the possibility of higher-period gaits (for example skipping [29]), an extended foot instead of a point foot [27], and other anatomical realism [27].

The simplest way of including a leg-swing cost would be to assume that it is a function of frequency and amplitude which is independent of gait. The legswing cost is then a function of $V$ and $D$, has no effect on which gait uses less energy at a given $V$ and $D$, and therefore has no effect on which gait is optimal at that $V$ and $D$. Figure 3 would be exactly unchanged. The simplest way of incorporating elastic recovery is to assume that a fixed fraction of the leg work is from elastic energy storage and hence should have no cost in the optimization. This would scale the costs of all gaits by the same constant (less than 1) and would therefore have no effect on any of the relative costs of various gaits. Thus, leg-swing and elastic-recovery effects can affect gait choice only through more complex dependences.

We do not know which neglected effects are the most important for explaining the deviations of observed human behavior from the model predictions here, particularly the prediction of the pendularrunning gait, which seems little used by humans. Nonetheless, this model, having no free parameters, might most simply explain why we choose walking and running over the plethora of other possible gaits.

\section{Methods}

\section{Formulation}

The governing equations are

$$
m \ddot{x}=F\left(x-x_{c}\right) / l, m \ddot{y}=-m g+F y / l
$$

for stance with duration $t_{s}$, and

$$
\ddot{x}=0, \ddot{y}=-g
$$

for flight with duration $t_{f}$, where $l=\sqrt{\left(x-x_{c}\right)^{2}+y^{2}}$ . Time $t=0$ is the beginning of a stance phase with foot-contact point $x_{c}=0$. The initial conditions are $x(0)=x_{0}, y(0)=y_{0}, \dot{x}=\dot{x}_{0}$ and $\dot{y}=\dot{y}_{0}$. At $t=$ $t_{f}+t_{s}$, periodicity requires that $x_{f}=x_{0}+d, y_{f}=y_{0}$, $\dot{x}_{f}=\dot{x}_{0}$ and $\dot{y}_{f}=\dot{y}_{0}$. The numerical integration then determines $v, d, l_{\max }$ and $C$. For given $l_{\max }, d$ and $v$, we seek the control strategy $\left(x_{0}, \dot{x}_{0}, y_{0}, \dot{y}_{0}, F(t), t_{s}\right)$ that minimizes the work-based specific mechanical cost of transport

$$
C=\int_{0}^{t_{s t e p}} \frac{[F(t) \dot{i}]^{+}}{m g d} d t
$$




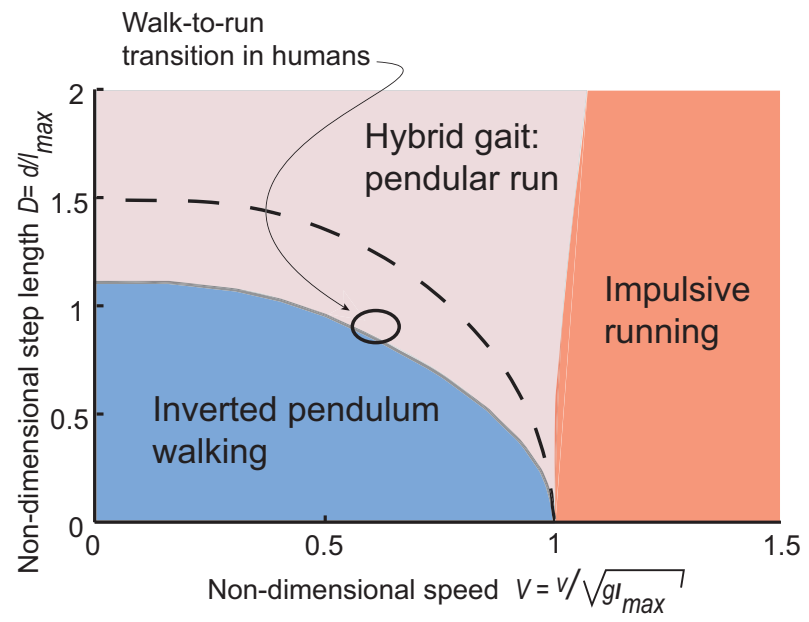

Figure 3: The regions in which each of the three collisional gaits are optimal. Inverted-pendulum walking ceases to be locally optimal at the pendular-run interface. The oval indicates the approximate speed and step length range at which humans switch from walking to running [18; 19]. The dashed line indicates where compression-only inverted-pendulum walking becomes mechanically infeasible (typically approximated [8] as $V=1$, which is correct for small $D$ ). At the right part of the intermediate region, the pendular run is almost impulsive running; at the left edge, it is almost inverted-pendulum walking.

where []$^{+}$is non-zero only for positive values $\left([p]^{+}=p\right.$ if $P=0$ and $[p]^{+}=0$ if $\left.p<0\right)$. The only cost is for mechanical work $(d W=F d l)$.

\section{Numerical solution of the optimal control prob- lem}

We non-dimensionalize all quantities by $l_{\max }, M$ and $g$. We seek

$$
\begin{aligned}
& \left(X_{0}, \dot{X}_{0}, Y_{0}, \dot{Y}_{0}, \bar{F}(\tau), \tau_{s}\right)= \\
& \quad\left(x_{0} / l_{\max }, \dot{x}_{0} / \sqrt{g l_{\max }}, y_{0} / l_{\max },\right. \\
& \left.\quad \dot{y}_{0} / \sqrt{g l_{\max }}, F(t) / m g, t_{s} \sqrt{g l_{\max }}\right)
\end{aligned}
$$

where $\tau$ is the non-dimensional time, that produce the optimal periodic gait with given $V$ and $D$, and with the non-dimensional step-length satisfying $0 \leq L(\tau) \leq 1$.

The infinite-dimensional search space for this optimization problem contains the set of all possible functions $\bar{F}(\tau)$. We restrict our search to the set of piecewise linear functions, defined on an evenly spaced time-grid $\left(0=\tau_{0}, \tau_{1}, \tau_{2}, \ldots, \tau_{N}=\tau_{s}\right)$, with grid spacing ${ }^{4} \tau_{i}-\tau_{i-1}=h=\tau_{s} / N$. So the search space becomes $z=\left(X_{0}, \dot{X}_{0}, Y_{0}, \dot{Y}_{0}, \bar{F}_{i=0 \ldots N}, \tau_{s}\right)$, where $\bar{F}_{i}=\bar{F}\left(\tau_{i}\right)$. The linear constraints are $\epsilon \leq \tau_{s} \leq \tau_{\text {step }}$, $\bar{F}_{\text {min }} \leq \bar{F}_{i} \leq \bar{F}_{\text {max }}$. We need $\epsilon>0$ because a periodic step requires a stance phase. In addition, although the forces are allowed to be unbounded conceptually, for numerical optimization they need to be bounded: we choose a bound $\bar{F}_{\max }>>1$ and $\bar{F}_{\min }=0$. Ultimately $\bar{F}_{\max }$ is allowed to grow arbitrarily, so that it is not a parameter in the solutions we present. Interestingly, choosing $\bar{F}_{\text {min }}<0$, allowing tensional legforces, does not affect the optima. The leg-length constraint, $0 \leq L(\tau) \leq 1$, is enforced at the grid points $\tau=\tau_{i}$. Gait periodicity is another nonlinear constraint.

For given $z, C$ and the constraint violations are evaluated by integration of the differential equations. $C(z)$ is to be minimized subject to the various linear and nonlinear equality and inequality constraints: $g_{\text {eq }}(z)=0$ and $g_{\text {ineq }}(z) \leq 0$. We smooth $C(z)$ with $h$ as a smoothing parameter. We used a particularly robust implementation of Sequential Quadratic Programming (SQP) [30] for the optimization.

Convergence to the idealized collisional gaits is discovered by letting $N \rightarrow$ large, $\bar{F}_{\max } \rightarrow$ large and $\epsilon \rightarrow$ small. At high $V$, if $\bar{F}_{\max }$ is set large enough for a given $\epsilon, \bar{F}_{\max }$ has no effect on $C$. The optimization then always finds $\tau_{s}=\epsilon$ as $\epsilon \rightarrow 0$, thus converging to impulsive running. We assure ourselves of the convergence to the collisional walking by Richardson extrapolation. That is, we solve the problem for grids of sizes $N=N_{1}, N_{2}, N_{3}, \ldots$ assuming that the cost is a smooth function of $N^{-1}$, and extrapolating the cost to $N^{-1} \rightarrow 0 . \bar{F}_{\text {max }}$ is maintained high enough and $\epsilon$ low enough to be unused constraints. The ODE solutions are accurate to about $10^{-14}$ over a grid interval (obtained by integrating from grid-point to grid-point with

\footnotetext{
${ }^{4}$ The published version had $\tau_{i}-t_{i-1}=h=\tau_{s} / N$, a typo

${ }^{5}$ The published version had $\bar{F}_{i}(\tau)=\bar{F}\left(\tau_{i}\right)$, a typo.
} 


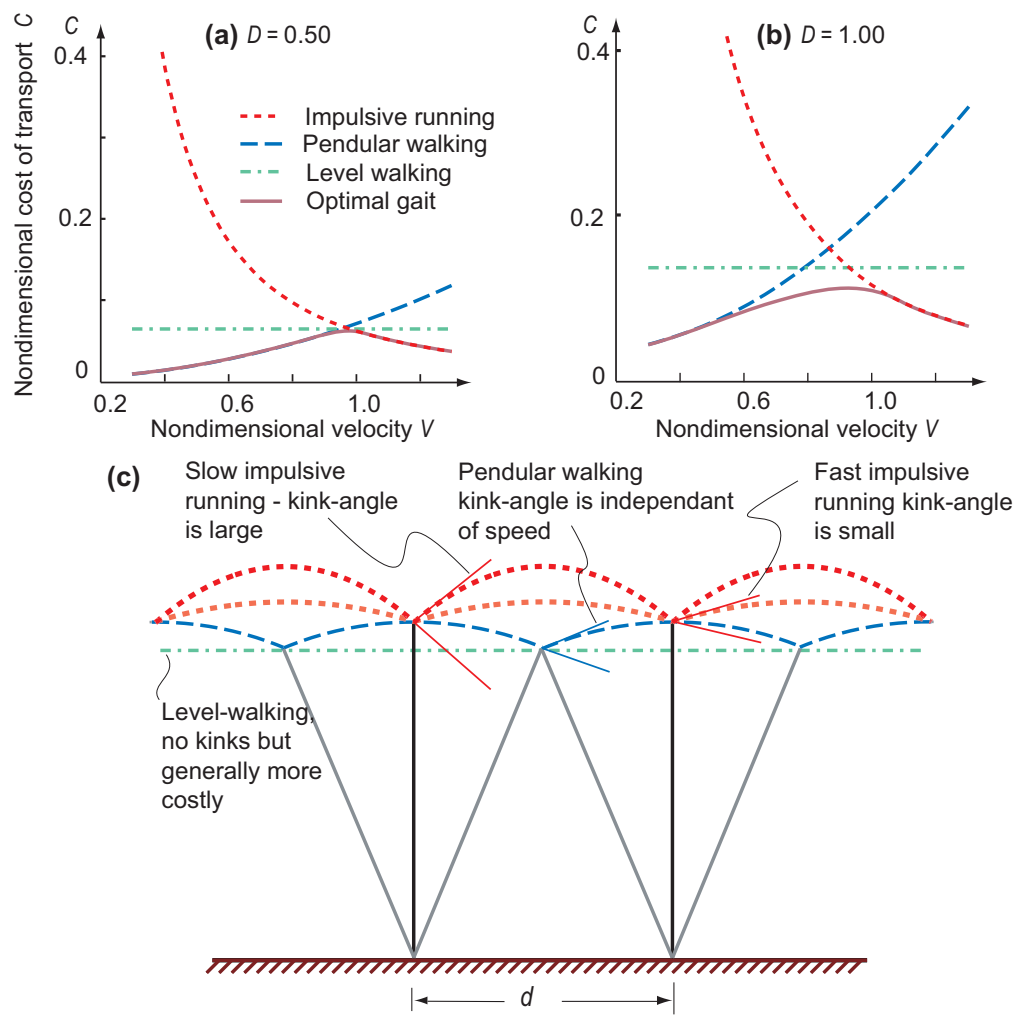

Figure 4: Cost of transport versus speed. a, For small $D(=0.50)$, all periodic gaits (that do not involve leg tension) have nearly equal costs near $V=1$. Inverted-pendulum walking is optimal at low speeds, pendular running at a narrow range of intermediate speeds, impulsive running at high speeds, and flat walking is never optimal. b, However, for large $D(=1.00)$ and for $V \approx 0.8-0.9$, flat walking, perhaps like a 'Groucho walk' [25], although not optimal, has lower cost than both inverted-pendulum walking and impulsive running. The colors used in a and b indicate the following gaits: red, impulsive running; blue, pendular walking, green, level walking; purple, optimal gait. c, Body trajectories for a pendular walking gait (blue; kink angle is independent of speed), a low-speed impulsive running gait (red; kink angle is large), a high-speed impulsive running gait (orange; kink angle is small) and level walking (green; no kinks, but generally more costly), all with the same step length.

an adaptive RK-45 method, benchmarked by a Taylorseries method) and accurate to less than $10^{-14} \mathrm{~N}$ over the whole step. We thus avoid significant sources of error not related to the finiteness of $N$ and can therefore treat the convergence as dependent only on $N$. The convergence is observed to be linear in $N^{-1}$. The linearly extrapolated limit of the sequence of $C$ values is found to differ from the cost of the corresponding analytically determined inverted-pendulum collisional walking gait by a relative error of about $10^{-3}$.

For each $V$ and $D$, multiple optimization runs, each started with a different initial seed, all converged towards the same control strategy, indicating the likely uniqueness and globality of each collisional minimum. To determine the regions in which each gait is optimal more precisely (Fig. 3) we repeated the optimization over the space of (analytically calculable) collisional gaits.

\section{Pontryagin's maximum principle}

Pontryagin's maximum principle [31] can be used over the stance phase, neglecting the leg-length constraint, to get necessary conditions on the optimal solutions. This calculation shows that during stance, if the optimal control is not singular, the leg-forces must be maximum $\left(F_{\max }\right.$, apparently corresponding to heel-strike or push-off), or zero (stance simulating flight by having no force). This much agrees with our full optimizations and heuristics. The pendular stance portions we found, with $i=0$, seem to be singular arcs of the optimal control.

\section{References}

[1] J.A. Borelli. On the movement of animals (De Motu Animalium, Pars prima). P. Maquet (trans.), 1989. Springer-Verlag, Berlin, p. 152., 1680.

[2] R. Margaria. Biomechanics and Energetics of 
Muscular Exercise. Oxford, UK, Clarendon Press., 1976.

[3] D. F. Hoyt and C. R. Taylor. Gait and the energetics of locomotion in horses. Nature, 292:239240, 1981.

[4] R. McN. Alexander. Optimum walking techniques for quadrupeds and bipeds. J. Zool., Lond., 192:97-117, 1980.

[5] R. McN. Alexander. Optimization and gaits in the locomotion of vertebrates. Physiol. Rev., 69:1199-1227, 1989.

[6] R.McN. Alexander. A model of bipedal locomotion on compliant legs. Phil. Trans. R. Soc. Lond., B338:189-198, 1992.

[7] A.E. Minetti and R. McN. Alexander. A theory of metabolic costs for bipedal gaits. J. Theor. Biol, 186:467-476, 1997.

[8] R. McN. Alexander. Mechanics of bipedal locomotion., volume 1, page 493504. Pergamon Press, New York, 1976.

[9] G. A. Cavagna, N. C. Heglund, and C. R. Taylor. Mechanical work in terrestrial locomotion: two basic mechanisms for minimizing energy expenditure. Am. J. Physiol., 233:243-261, 1977.

[10] R. McN. Alexander. Principles of animal locomotion. Princeton University Press, Princeton., 2003.

[11] A. D. Kuo. Energetics of actively powered locomotion using the simplest walking model. $J$. Biomech. Eng., 124:113-120, 2002.

[12] A Ruina, J.E. Bertram, and M. Srinivasan. A collisional model of the energetic cost of support work qualitatively explains leg-sequencing in walking and galloping, pseudo-elastic leg behavior in running and the walk-to-run transition. $J$. Theor. Biol., doi: j.jtbi.2005.04.004, 2005.

[13] A. D. Kuo, J. M. Donelan, and A. Ruina. Energetic consequences of walking like an inverted pendulum: step-to-step transitions. Exer. Sport Sci. Rev., 33:88-97, 2005.

[14] N. Rashevsky. Studies in the physicomathematical theory of organic form. Bull. Math. Biophysics., 6:1-59, 1944.

[15] R. McN. Alexander. Elastic mechanisms in animal movement. Cambridge University Press, Cambridge., 1988.
[16] T.A. McMahon and G.C. Cheng. The mechanics of running: how does stiffness couple with speed? J. Biomech., 23, Suppl.1:65-78, 1990.

[17] A. D. Kuo. A simple model predicts the step length-speed relationship in human walking. Journal of Biomechanical Engineering, 123:264269, 2001.

[18] A. Thorstensson and H. Robertson. Adaptations to changing speed in human locomotion: speed of transition between walking and running. Acta. Physiol. Scand., 131:211-214, 1987.

[19] A. E. Minetti, L. P. Ardigo, and F. Saibene. The transition between walking and running in humans: metabolic and mechanical aspects at different gradients. Acta Physiol. Scand., 150:315-323, 1994.

[20] R. McN. Alexander. Work or force minimization as a criterion for the evolution of locomotion. In Fourth World Congress of Biomechanics, Calgary, Canada, August 2002.

[21] J. D. Ortega and C. T. Farley. Minimizing center of mass vertical movement increases metabolic cost in walking. J. Appl. Physiol., 2005, doi:10.1152/japplphysiol.00103.2005.

[22] K. Gordon, D. Ferris, and A. Kuo. Reducing vertical center of mass movement during human walking doesnt necessarily reduce metabolic cost. In Proc. 27-th Annual Meeting. American Society Biomechanics, Toledo, $\mathrm{OH}$. American Society of Biomechanics, 2003.

[23] R. Blickhan and R. J. Full. Similarity in multilegged locomotion: bouncing like a monopode. $J$. Comp. Physiol. A., 173:509-517, 1993.

[24] D.M. Bramble and D.E. Lieberman. Endurance running and the evolution of homo. Nature, 432:345-352, 2004.

[25] J.E.A. Bertram, P. DAntonio, J. Pardo, and D. V. Lee. Pace-length effects in human walking: groucho gaits revisited. J. Motor Behavior., 34:309318, 2002.

[26] R. L. Marsh, David J. Ellerby, Jennifer A. Carr, Havalee T. Henry, and Cindy I. Buchanan. Partitioning the energetics of walking and running: swinging the legs is expensive. Science, 303:8083, 2004. 
[27] Anderson F. C. and Pandy M. G. Dynamic optimization of human walking. Journal of Biomechanical Engineering, 123:381-390, 2001.

[28] R.McN. Alexander. Invited editorial on "interaction of leg stiffness and surface stiffness during human hopping”. J. App. Physiology, 82:13-14, 1997.

[29] A. E. Minetti. The biomechanics of skipping gaits: a third locomotor paradigm? Proc. R. Soc. B, 265:1227-1235, 1998 .

[30] P. E. Gill, W. Murray, and M. A. Saunders. Snopt: An sqp algorithm for large-scale constrained optimization. SIAM J.Optim., 12:9791006, 2002.

[31] A.E. Bryson and Y.C. Ho. Applied Optimal Control. John Wiley, NY, 1975.

The published version incorrectly reversed the author order of [21]. 